\title{
An overview and classification of research approaches in green wireless networks
}

\author{
Luis Suarez ${ }^{*}$ Loutfi Nuaymi and Jean-Marie Bonnin
}

\begin{abstract}
Energy consumption of wireless networks is now a very important research topic and several research teams worldwide are proposing solutions for the so-called green wireless networks, i.e. energy-efficient wireless networks. Although the increase of this research activity is rather recent, a great number of research papers and collaborative projects exist nowadays. We first summarise the metrics used in the related literature for performance evaluation. Then, we focus on describing the current approaches proposed by reviewing a good number of references from literature. The main research directions are presented: the component level research, where the efforts are mainly concentrated on the power amplifier section; the cell layout adaptation including the cell-breathing technique and coverage extension methods like femtocells and relays; in addition, we also include the radio resource management and the cognitive radio into the studied approaches. These methods are analysed, compared, classified and then a framework of classification and integration is proposed. We finally describe some major collaborative projects dedicated to this topic.
\end{abstract}

Keywords: green radio, energy efficiency, wireless networks.

\section{Introduction}

The constant advances in the world of wireless communications bring to end-users new services and features never expected in the past. Nevertheless, they also bring new challenges and issues to overcome. A major problem to solve is the quick growth of energy consumption in the Information and Communications Technologies (ICT) infrastructure. Mobile and other wireless communications are great contributors to the rapidly increasing rate of ICT traffic due to the innovative services supported by the latest mobile platforms, increasing every day the energy consumed. According to the often-cited Gartner report [1], the ICT Market contributes 2\% of global GHG, Greenhouse Gases $\left(\mathrm{CO}_{2}\right)$ emissions. This contribution will rapidly grow if no immediate measures are taken. The first problem of the high levels of energy consumption is that it is often necessary to use fossil fuel (e.g. diesel), which produces large amounts of GHG. According to SMART 2020 report [2] the amount of $\mathrm{CO}_{2}$ due to ICT was $151 \mathrm{MtCO}_{2}$ during year 2002, where $43 \%$ was due to the mobile sector, and during

\footnotetext{
* Correspondence: luis.suarezrivera@telecom-bretagne.eu

Telecom Bretagne, RSM Department, Rue de la Châtaigneraie 2, 35510 Rennes, France
}

2020 it is forecast to rise to $349 \mathrm{MtCO}_{2}$ emitted, with $51 \%$ of emissions from the mobile sector. These values are more detailed in Figure 1. Some other important problems are the energy bill (although less often mentioned by the operators), the energy availability in some underdeveloped areas, as well as the exposure to electromagnetic radiation due to mobile phone utilisation. Let us present some operators energy consumption levels here. In USA, the residential ICT market required 1\% (42 TWh) of the total energy produced in 2005 [3,4]; Telecom Italia during 2005 consumed around $2 \mathrm{TW}$-h corresponding to $1 \%$ of total Italian energy production [4-6]; also a similar figure of $2 \mathrm{TW}$-h was consumed by France Telecom in 2006 corresponding to $0.4 \%$ of the energy production of the whole country $[5,6]$.

The major part of the huge demands of energy comes from the radio access network (RAN), which represents $57 \%$ of the energy consumption of a cellular network [7] (see Figure 2). The high energy consumption of the RAN is due to two major reasons. First, the inefficient power supply conversion into transmission by base station (BS) systems, where the RF power amplifier section consumes between 60 and $70 \%$ of the energy supplied [8], dissipating a remarkable quantity of energy in terms of heat and 


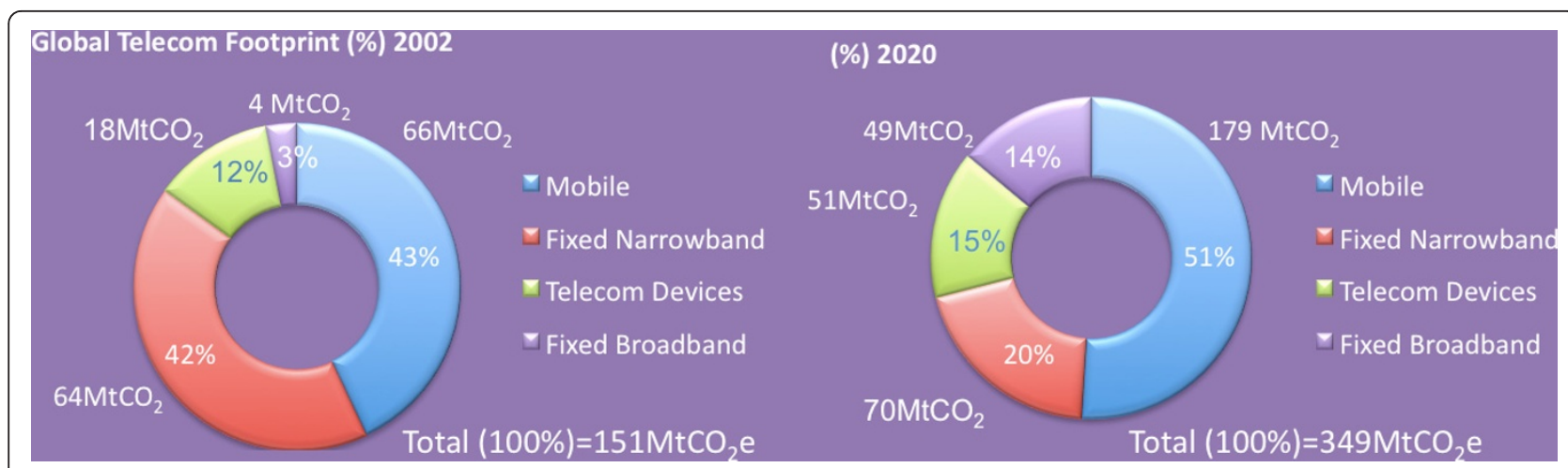

Figure 1 Carbon footprint contribution by Telecom for 2002 (left) and forecasted values to 2020 (right). Source: [2].

only a small fraction corresponds to the useful output. In Figure 3, we present a synthesis we made of different sources that shows the consumption contribution from different components $[7,9-11]$. The second reason is the fact that traffic in the real world cannot be considered uniform, neither geographically nor temporally speaking, wasting therefore an important percentage of energy due to unnecessary active BSs. In [6], it is mentioned that for a typical radio access deployment, only $10 \%$ of BSs are responsible for nearly a half of the traffic, whereas $50 \%$ of the BSs handle $5 \%$ of total traffic. Taking into account this panorama, researchers around the world are looking for ideas to solve or at least mitigate such problematic.
These ideas include new architectures and mechanisms at different levels (i.e. internal components, BS, RAN), some of them conceived into the core of the already important list of international projects working on wireless network energy efficiency.

The overview we propose in this article is definitely not the first on this topic. The concerns about energy efficiency are something that quickly grows worldwide. We can say that the last decade was a period of progressive awakening and rapid awareness on the subject. To the best of authors' knowledge, a first survey on the topic was published in the year 2001 [12]. This survey explored the different advances and research approaches for wireless

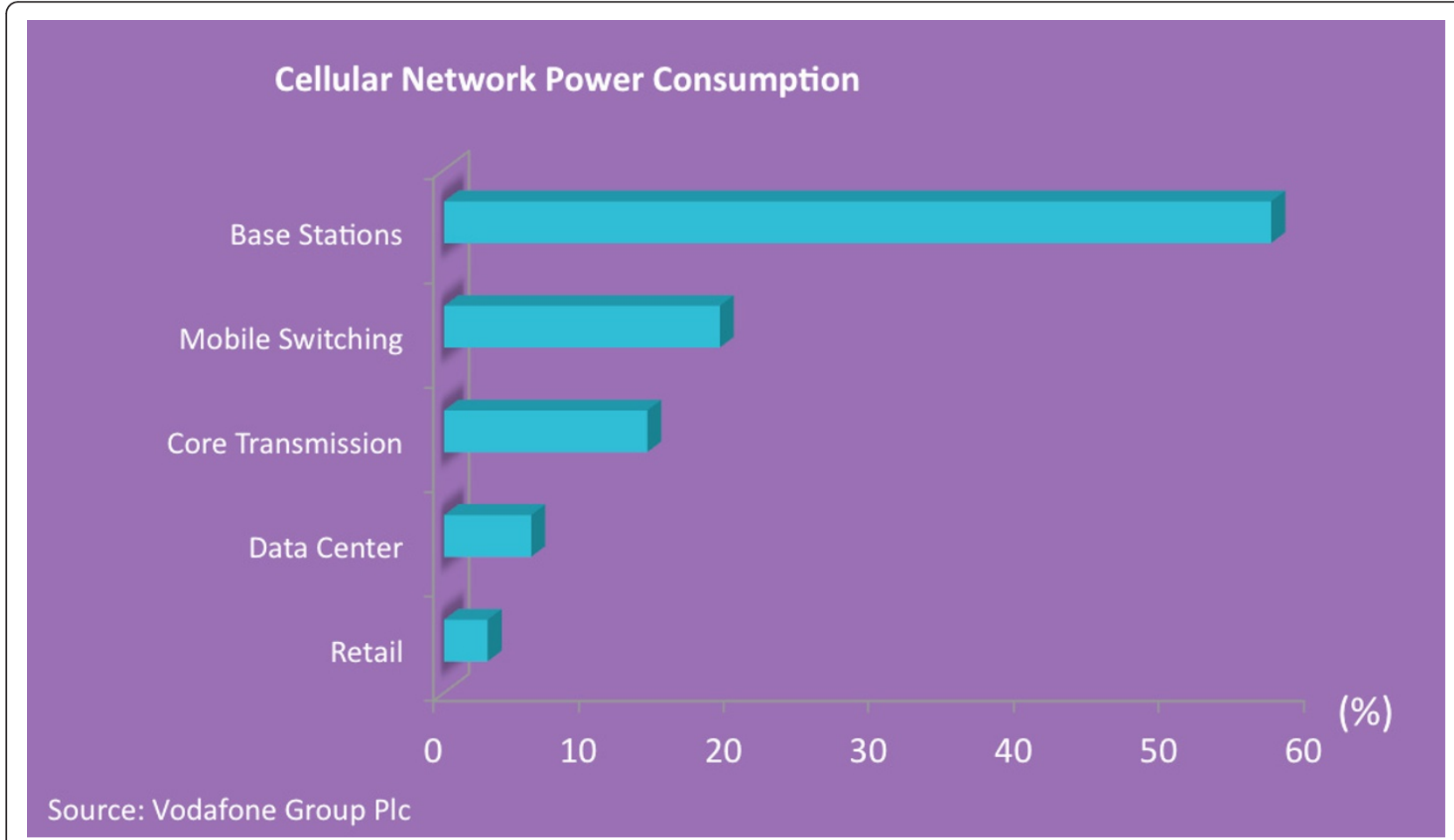

Figure 2 Percentages of power consumption into the cellular network infrastructure. Source: Vodafone GroupPlc [7]. 


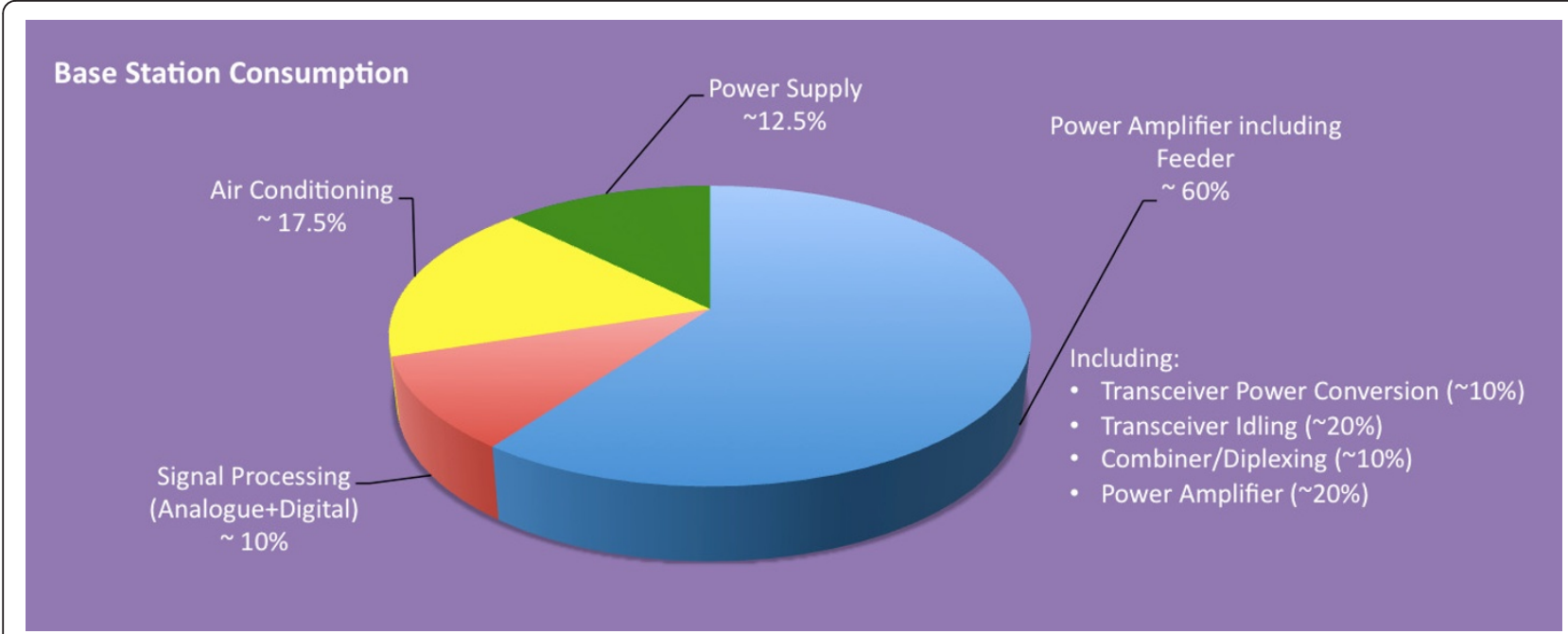

Figure 3 Estimated energy consumption in the BS. Synthesis of data from [7,9-11].

networks following each one of the layers of the wireless protocols stack going beyond the typical approach to physical layer, emphasising at that moment that higher savings could be achieved if also upper layer processes are optimised. Another survey in the domain of energy-efficiency techniques for mobile systems was proposed in 2004 [10] and it remains today a good entry point for this topic. This reference gives a wide overview of energy-efficiency mechanisms for wireless network, mainly WLAN and cellular networks, taking into account hardware, architectures and protocols. Some surveys were written in the framework of collaborative projects (see Section 8) such as [13] from the EARTH project or [14] from Mobile VCE Core 5 giving the future challenges and perspectives. A parallel overview of EARTH and Green Touch projects is presented in [15]. These surveys devote their work to describe what has been done so far within their projects, showing their own present and future steps of development. Some other surveys are dedicated to specific approaches like, for example, MIMO as presented in [16]. The authors of [17] focus on energy-efficient transmission, discussing the research on some techniques like MIMO, OFDM/ OFDMA, adaptive modulation, scheduling, etc. In this article, the radio resource allocation and transmission mechanisms are viewed from three different perspectives: space, frequency and time domain. The survey presented in [18] studies similar topics with [17] (e.g. MIMO and OFDMA); however, it includes a short discussion about relaying. We can also mention the exhaustive work of compilation and synthesis of [19] dedicated to the energy efficiency of fixed networks with an impressive list of more than 150 references.

In this overview, we synthesize the green wireless networks research trends and highlight the perspectives and futures challenges. The different approaches are presented by means of a describing the general principles and features of each one of them. The literature review has permitted us to compare the techniques based on the reported energy-efficient performance, consequences during network planning and operation and the future challenges. A framework of a classification and integration is also proposed. In this model, the energy-efficient techniques studied are arranged by means of a stacked structure of different layers. As we will see later on, in our presented model the lower layers serve the upper layers to increase their attainable energy-efficiency performance. The challenges, gaps between approaches and possible advantages of this integration are also discussed. Last but not least, we present a review of the projects being carried out by cooperation of governments, academic institutions, centres of research, vendors and telecommunication providers R\&D across the world in order to face the energy consumption problematic. Such projects attack the problem at different levels: the component enhancement with emphasis on power amplifier section and the reduction of internal losses; the architecture optimisation at the BS and network levels; the development of enhanced techniques on energy-aware radio resource management (RRM) and transmission schemes, and the research on cooperative, coordinated and selforganised mechanisms. What is more important is that the results of such projects will define the road to be followed by green networks in the years to come.

The structure of this article is as follows: in Section 2, we present a review of the metrics used in the related literature to measure the energy performance of systems and mechanisms. In Section 3, we discuss some mechanisms that reduce energy consumption, which fall into the category of cell layout adaptation (CLA) techniques. In this category, we include the cell shaping algorithms 
(Section 3.1), where energy consumption is reduced by responding to the incoming traffic pattern by means of switching off unnecessary BSs and adapting the sizes of remaining cells in order to guarantee coverage; we also give attention (Section 3.2) to describing the joint energy-efficient macro/femtocell deployments and their contributions in energy saving; finally, also in the category of CLA, we describe the wireless relaying techniques (Section 3.3) and how they can be a key tool for energyefficient transmission, both for downlink and uplink transmissions. Section 4 focuses on energy-aware RRM mechanisms. The cognitive radio (CR) technique as used in this framework is addressed in Section 5. Completing the description of solution approaches, Section 6, describes what has been done at the component level in order to optimise the internal architectures and components, which focuses mostly on the power amplifier. We present a synthesis of the techniques mentioned from the previous sections in Section 7 giving a comparison of approaches and proposing a classification and integration model of the existing energy-efficient techniques. A discussion of challenges, gaps and advantages of the integration of approaches is given. Finally, the most relevant collaborative projects devoted to the research on the wireless network energy consumption are described in Section 8 highlighting their goals and scope. Finally, the conclusions are given in Section 9.

\section{Metrics for energy-efficiency and consumption performance estimation}

Several metrics have been defined so far in order to characterise wireless network energy-efficiency and consumption at different levels, i.e. the internal components, the BS or the RAN, as already proposed in [20]. Such metrics may additionally be classified as energy-efficiency metrics or energy consumption metrics as briefly mentioned in [21]. An energy-efficiency metric corresponds to the ratio of attained utility (e.g. transmission distance reached, area covered, output power, bits transmitted, etc.) to the consumed power/energy used. On the other hand, an energyconsumption metric corresponds to the energy/power consumed per unit of attainable utility. In Figure 4, we provide a classification of metrics using these already discussed criterions. The purpose of this section is to summarise the main metrics used in the literature to quantify the energy efficiency/consumption of physical devices and techniques aimed to this purpose.

Some relevant metrics concerning the component level are discussed in the following. Important attention is given on the power amplifier efficiency metric (i.e. ratio of PA output power to supplied power) [20], due to the already-mentioned lack of performance of this element. Some other metrics are also considered for power amplifier section like the peak-to-average power ratio (PAPR).
The reduction of the PAPR guarantees better amplifier efficiency $[13,22]$. However, the interest is not only centred on the power amplifier. Other sections of the RF transmission chain, where any improvement in terms of efficiency counts (e.g. antenna elements), are also considered. Nevertheless, all the efforts are not devoted to the analogue components and the digital section is also taken into account. Some specific metrics have been used so far in order to measure the performance of computing processing associated to energy consumption (e.g. MIPS/Wmillions of instructions per second per watt; MFLOPS/ $\mathrm{W}$-millions of floating point operations per second per watt) [20]. Also at this layer we can include the energy consumption gain [14] that corresponds to the ratio of the consumed energy of a baseline device to the consumed energy of a given device under test. This metric may be extended from separated components like a new power amplifier prototype to larger devices like BS systems.

At access node level, more precisely the BS, there is also a rich set of metrics. Although some classic metrics are still useful (e.g. average BS consumed power [20]), new metrics have specifically been introduced for this topic. The energy consumption rating (ECR) $[14,20]$ gives the energy used for transmitting a piece of information (Joules/bit) [14]. Some other metrics aim to observe the attained utility of the different resources regarding there exists trade-offs, such as the spectral efficiency $(\mathrm{b} / \mathrm{s} / \mathrm{Hz})$ and the power efficiency $(\mathrm{b} / \mathrm{s} / \mathrm{Hz} / \mathrm{W})$ [23]. One metric targeted to cover all the aspects in a more general way is the radio efficiency $((\mathrm{b} \cdot \mathrm{m}) / \mathrm{s} / \mathrm{Hz} / \mathrm{W})$ [23], measuring the data rate transmitted and transmission distance attainable given the respective figures of bandwidth and supplied power resources.

There are some other metrics that address the RAN performance. The metrics here evaluate the global attained service provision given a consumed power. This level of service provision can be measured as proposed by ETSI for GSM networks $[20,24]$, whether in terms of the ratio of subscribers served or the covered area to site power consumption. The ratio of number of subscribers served during the peak traffic hour to site power consumption is used for urban environments, whereas the ratio of area coverage attained to site area consumption is used for rural areas. In $[13,21]$, they propose to use as metric the consumed power per area unit $\left(\mathrm{W} / \mathrm{m}^{2}\right)$. According to [21], in order to avoid misinterpretations it is important to fix the coverage area for having then a point of reference for comparisons.

\section{Cell layout adaptation}

In this section, we will speak of the techniques in the category of CLA. This category, as we will see in Section 7, it is on the top of our classification and integration model. 


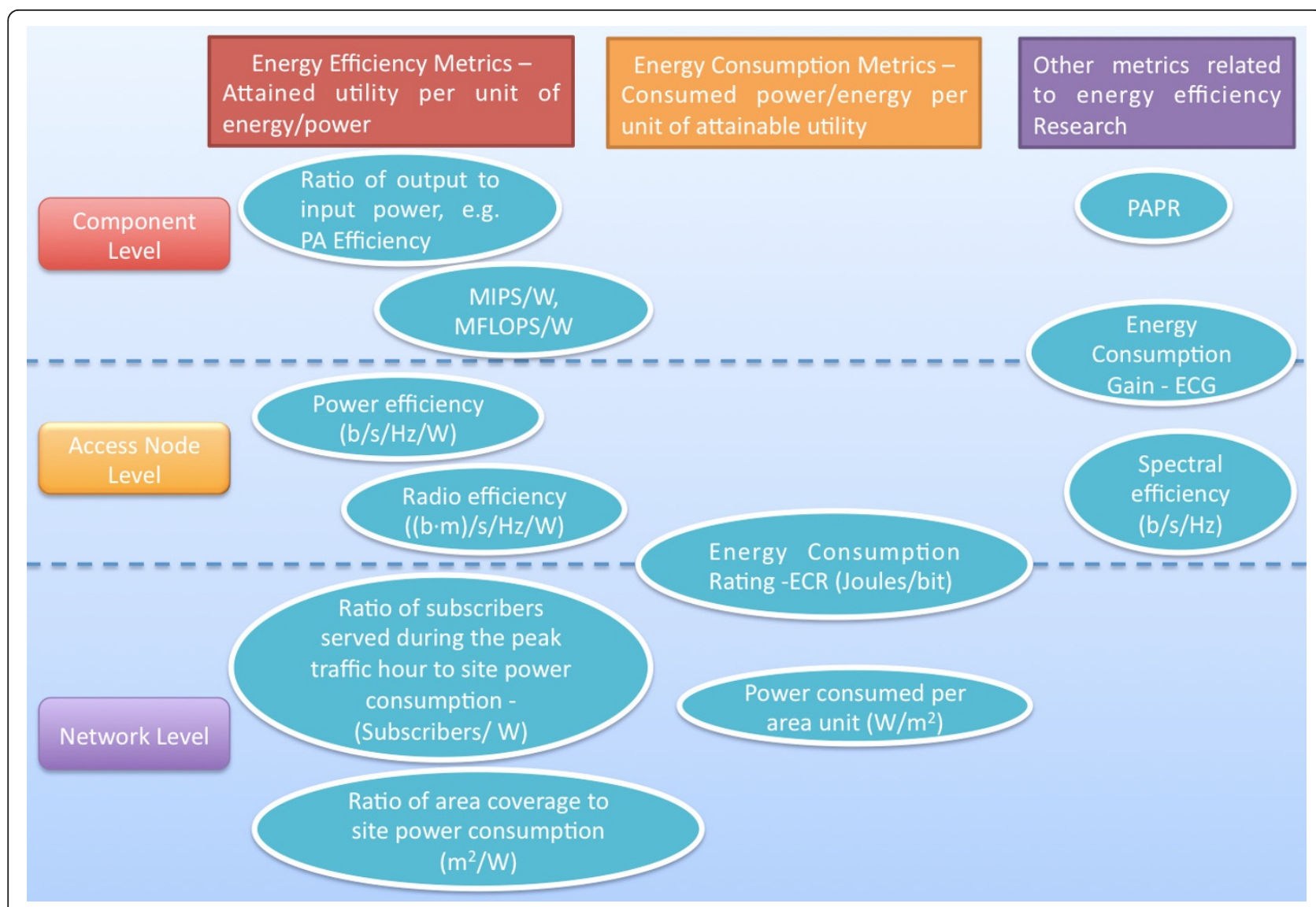

Figure 4 Classification of metrics used in energy efficiency for wireless networks. Synthesis of sources from [13,14,20-24].

The reason for this is the fact that approaches from this category yields the higher energy reductions at a network scale. However, these top layer mechanisms must rely on lower layer approaches to enhance their savings. The techniques from lower layers have a repercussion in a more reduced scale such as the BS transmission performance or the internal component level savings. The techniques into the category of CLA allow adapting, customising and extending the infrastructure of the RAN in function of spatial and temporal traffic distributions, aiming to reduce the network energy consumption. In this category, we consider the cell shaping techniques (i.e. BSs switching-off and cell breathing), the macro-femtocell deployments and last but not least, the relays.

\subsection{Cell shaping techniques}

A first way to globally reduce the energy consumption in a cellular network is to adapt the cell layout to the traffic distribution by cell shaping (see Figure 5). In such a category, we introduce the switching-off and cell-breathing schemes. In a basic switching-off and cell-breathing mechanism, the idea is to turn off, or equivalently send to the sleep mode state, the most number of BSs during the low traffic period, with no compromise of coverage or service availability. Here, a blocking probability threshold must be respected. Additionally, the cells kept active must take charge of the remaining traffic, needing to increase their coverage range constrained by a maximum BS transmission power and therefore the cell is limited to a maximum coverage radius. In order to perform the switching off, it is necessary that the BSs about to be deactivated redistribute the traffic to their closest neighbours. Some BSs will not be able to redistribute their full traffic and will remain active with a lower transmission power. The dynamic of cells has a size adaptation to incoming traffic, that metaphorically speaking can be seen as the cell is breathing.

The switching-off and cell-breathing literature can be classified into two types: switching-off network planning and cell-breathing coordination. Firstly for network planning, in [25], a switching-off planning method is proposed. This method allows finding the ratio of deactivated BSs to remaining active BSs, as well as the switching-off period, where traffic is considered sufficiently enough low to perform the BS switching-off not violating a blocking probability limit. They call this low traffic period the "night 


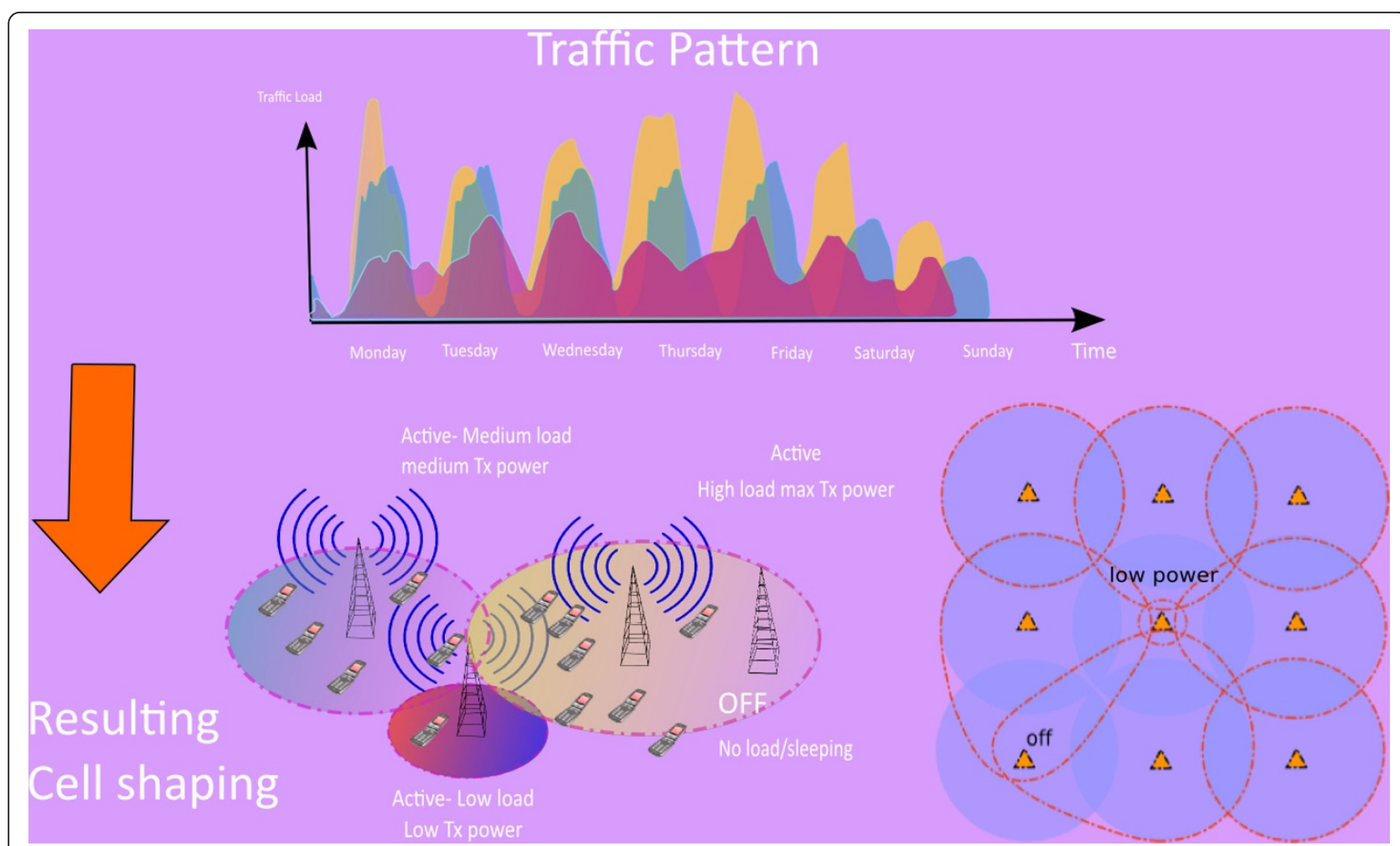

Figure 5 Cell shape adaptation in function of traffic distribution. The traffic pattern is the main input, which permits to shape the cell deployment, i.e. switching off the BSs or changing transmission power levels.

zone" In [26], the authors from [25] present a generalised method that permits to calculate and optimise the night zone period independently of the existing cell geometry architecture.

There are other references that devote their efforts on proposals for dynamic cell-breathing coordination. The study of [27] proposes to concentrate the network traffic preferring highly loaded BSs, whereas remaining idle BSs can be switched-off. This reference takes into account also the importance of keeping a balance in the spectrum efficiency versus energy efficiency trade-off. For this purpose, the BS-MS association algorithm does not neglect the spectrum efficiency importance and gives preference to the associations that yields the best possible spectrum efficiency values. This article presents a distributed and a centralised version (i.e. a central device that provides coordination) of the cell-breathing algorithm. The results presented show that, despite the fact the distributed version exhibits a lower signalling overhead, the centralised version presents higher energy efficiency. The work presented in [27] can be considered as a preliminary version of the cell zooming approach proposed in [28]. For this reference also a centralised (i.e. the coordination role is played by the cell zooming server) and distributed versions of the algorithm are presented. The most remarkable advance compared to [27] appears in the centralised version proposed. Here, the algorithm is executed in two phases: In a first phase, the cell zooming server associates all MSs and BSs preferring the associations where the attainable spectrum efficiency is the best among the different association possibilities. Afterwards, any BS whose load is zero can go to sleep. In a second phase, the cell zooming server redistributes traffic from very low loaded BSs to the top highly loaded ones. By doing this, some new BSs may also be switched off. There is a continuation of the work about cell zooming in [29]. Here, the authors discuss the network planning issues in order to enhance a cellular deployment to better exploit the cell zooming technique. This article analyses the efficiency improvements due to smaller cell deployments and proposes to continue the work toward coverage extensions techniques such as relays. However, the cell zooming proposal is not the unique algorithm of its type. The algorithm presented in [30] combines the cell breathing with a tilt angle optimisation algorithm. Into this algorithm, each BS decides to become a candidate to be switched off if the BS is below a low traffic threshold. A centralised node is in charge of sequentially deactivating the BS candidates to go to sleep mode. This sequentiality allows evaluating the consequences of switching off a given $\mathrm{BS}$. The algorithm continues till there are not candidates to switch off. Moreover, in [31], a cell-breathing 
style algorithm with a new ingredient known as protocooperation is proposed. The proto-cooperation is a term originally used in biology and it refers to an interaction among species where nobody is mutually getting benefit of such an interaction. The authors of [31] propose the proto-cooperation for mutual collaboration of BSs deployed to reduce energy consumption. This algorithm as well as [30] uses traffic thresholds but with the difference is a fully distributed BS-based algorithm. On the other hand, in [32] another concern into the domain of cell-breathing coordination is analysed. In this article, soft transitions to pass from active to sleep mode and vice versa are proposed in order to avoid jeopardising the network during the traffic redistributions. It is shown here that such transitions can be achieved in a very short period of time that it is not going to significantly affect the energy efficiency expected from the cell-breathing technique.

There are several things to work in the future into this approach. From the literature review, we see a good number of publications propose algorithms for switching-off and cell breathing. However, there is still work to do in the transition of the network states and handover as we saw in [32]. Moreover, although there is already some work done into the aspect of joining the cell-breathing approach with coverage extension techniques (CET) such as femtocells and relays, this work is still in the first stages of development. We will discuss about femtocells and relays in the next two sections.

Future proposals on cell-breathing coordination must delve deeper into things like the feasibility of such algorithms to be implementable into a large-scale network. It is known that centralised coordination approaches yield better performance results than distributed proposals. The clear advantage of the centralised approaches lies on the fact that central nodes have a full view of network and better decisions can be made. However, to depend on centralised nodes can limit the network scalability, as well as introducing single point failure nodes. One clear direction is to find better BS-based distributed algorithms based on cooperative approaches where the main objective is the overall savings and not the local benefit into the BS. A halfway solution between the centralised and distributed approach could be the utilisation of clusters dividing the network and distributing coordination responsibility.

\subsection{Macro-femto radio access deployments}

Also in the same category of CLA we find the hybrid deployments of macro/femtocells (see Figure 6), a technique that is gaining more and more relevance into the current trends. We consider this approach as a future way of enhancement of the cell shaping techniques already discussed in Section 3.1. Let us remark that the utilisation of smaller cells but denser deployments combined with cell breathing is something already presented by Weng et al. [29]. The consequences of joint macro/ femtocell deployments are something deeply discussed in [8]. As it is well known, a femtocell is achieved by using a small BS (or access point) with coverage of tens of metres (see, e.g. [33]). The literature mentions the advantages of these technologies like lower energy consumption and low maintenance costs compared to those of a macroBS $[33,34]$, as well as higher dedicated data rates and better QoS for small groups of users levels, which leads to better user perception [8,33]. In [8], the benefits of using a joint macro/femto deployment are studied. The results have shown that femtocells are a good solution in order to enforce the capacity of a macrocell deployment without a high increase of energy consumption avoiding the introduction of new macroBSs to extend coverage and availability. In addition, this reference provides a discussion of introducing switching-off mechanisms combined with hybrid deployments. It is clear that if we increase the femtoBSs density too much it could turn out in overprovisioning that may lead the network to increase the consumption. In order to avoid this, the authors of [8] propose the utilisation of sleep modes to control the number of active femtoBSs, which permits to increase the femtoBS density enforcing coverage level without a significant impact on network energy consumption.

On the other hand, in [35], macro/femtocells deployments versus macro-only deployments are compared supported on real-life data and simulations. The simulation makes a forecast of energy consumption and total economic cost considering the IMPEX (implementation expenditure), OPEX (operational expenditure) and CAPEX (the capital expenditure, i.e. the expenditure associated to upgrades) for different strategies of macro/ femto deployment upgrades, year-by-year, throughout a period of 8 years. The results show that if the femtoBSs are deployed first during the year and then, according to necessities, macroBSs are added later, it gives as result a good strategy of network growth, because a higher number of femtoBSs installed at the beginning of the year reduces the necessity to install new macroBSs, which could impact more significantly the energy consumption. Nevertheless, the authors warn that an exaggerated growth rate of femtoBSs installed can affect, from an economic point of view, the network OPEX.

Additionally, the femtocell solution brings also some technical drawbacks and issues to overcome as for example the inter-cell coordination and interference. In [36,37], mechanisms for reducing the femto-macrocell interference by proper choices of bandwidth allocation are discussed. Both mechanisms propose in essence to divide a macrocell in two subregions: the inner region where the macrocell power dominates over deployed 


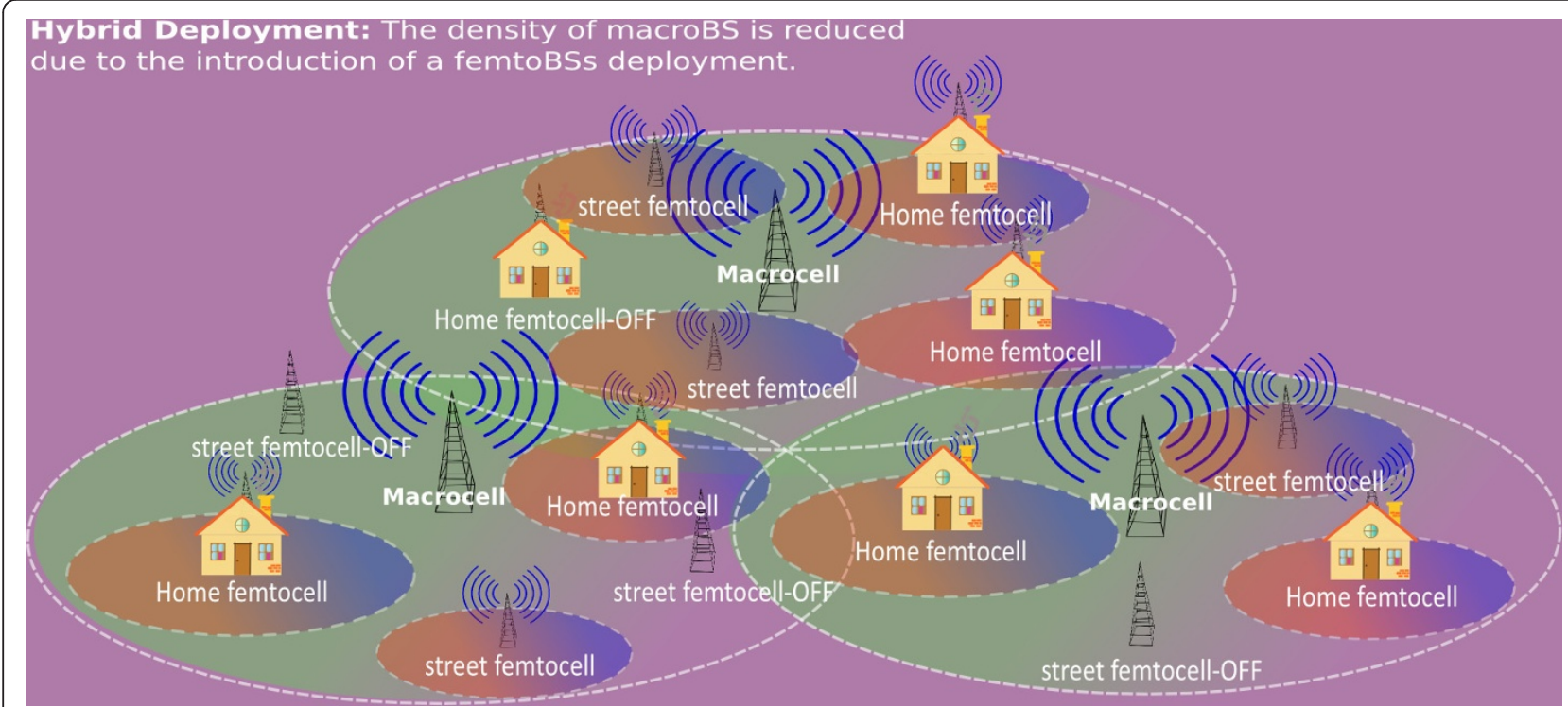

Figure 6 Simplified diagram of a joint deployment of femto/macro BSs

femtocells and the outer region where the femtocell dominates over mobile device uplink transmissions. The boundary of both regions is to be defined. For the inner region, it is proposed to use partitioned spectrum, where femtocells and macrocells use non-overlapping sections of the spectrum; for the outer region, the bandwidth is shared assuming that the macrocell transmission power does not overwhelm the femtocell power. In order to select the bandwidth usage mode, the ILCA (Interference-limited coverage area) of a femtocell is used in [36] and defined as "the area within the contour where the received power levels from $f B S$ and $m B S$ are the same". By knowing the ILCA, the area of influence of the femtoBS is known and then the decision of whether to use the shared or partitioned spectrum is made based on an area size threshold. In [37], uplink and downlink transmissions are handled differently and bandwidth usage mode depends on specific situations. In the downlink transmission, the femtoBS can select the bandwidth usage mode because the femtoBS is the interference victim given the higher downlink power of the macroBS. For uplink, it is up to the macroBS to decide the usage mode because farther MSs associated to the macroBS can be interference victims of outer femtoBSs.

The utilisation of cell breathing on hybrid networks remains as an open topic. Although it always exists the possibility of having improvements on femtoBS hardware, an important work remains still on the cell-coordination algorithms that permit the interaction of macro/ femtoBS in order to redistribute the network load in order to switch off any unnecessary device off. Those future algorithms must exploit the granularity that femtocell could bring to cover small spots uncovered or the capability of sharply adapting the access device resources to the density of users given in a zone. Thanks to femtocells and cell breathing we will achieve a fine-tuning of network coverage with an energy consumption tailored to the real necessities in the years to come.

\subsection{Relays}

A third approach in the category of CLA is the use of relays. This technique has been considered so far as a different alternative to the use of femtoBSs. Both can be considered as valid CET to enhance a deployment by increasing its energy-efficiency performance. Based on the references used in this section, we can say that relaying can be performed in two ways: (i) using repeater stations or (ii) using the mobile stations as relays. We can consider reference [38] into this first approach of using dedicated devices as repeaters. Here, the use of relays is proposed in order to reduce the mobile phone transmitted power, which reduces therefore the radiation exposure of the user during the call. A network device named green antenna is presented (see Figure 7). These antennas are connected by a wired link (e.g. optical fibre) to the $\mathrm{BS}$ and have only receiver capabilities. With this approach, it is possible to increase the $\mathrm{BS}$ receiver range and therefore reduce the power levels needed by mobile phones for uplink transmission. It can be noticed here that by reducing the transmission power for uplink transmission, it implies that the mobile terminal reduces its battery energy consumption, which is also a contribution to overall energy savings.

Some other references propose the use of mobile phones as relays. In [39], a cooperative relaying mechanism is proposed under a modified Vickrey auctioning 


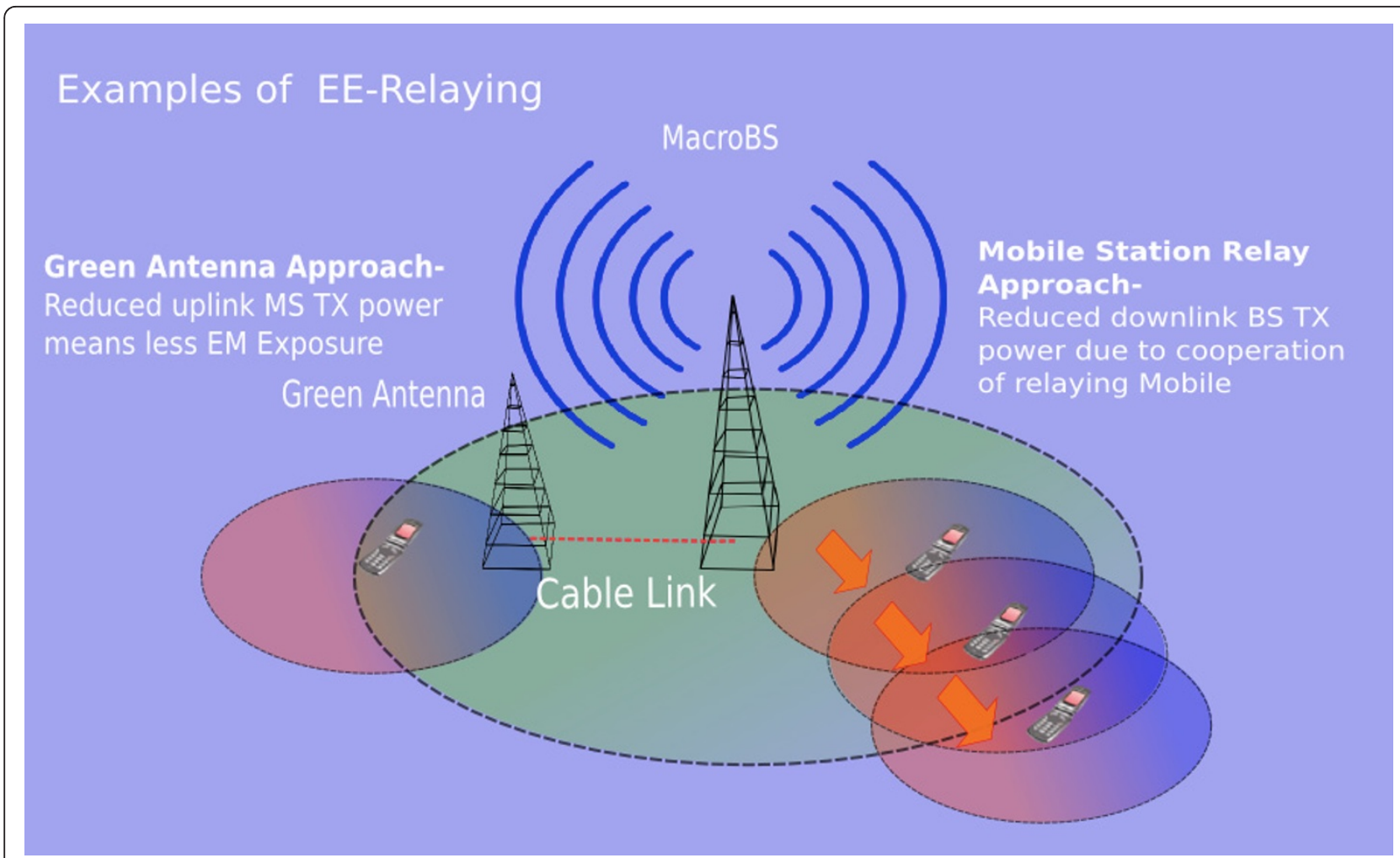

Figure 7 Two examples of relaying in the same figure: (left) a green antenna relay [38]; (right) downlink relaying using MSs as relays nodes [39].

strategy where a mobile phone serves as a relay for downlink transmission trading the expended energy on relaying for an economic reward. The reason for using a Vickrey model as the auctioning model is that the bidders, given the mechanism rules, are obliged to give a real value to the goods they trade [39], which allows a fair exchange. As we can see, this mechanism permits to reduce the transmission energy needed for the BSs systems that as we know is the most critical device into the mobile network in terms of consumption. Other references, on the contrary, give proposals but applied for the uplink. In [40], it is proposed to use close terminals to transmit data cooperatively to a common BS. When a given user is transmitting, a close idle terminal may decide to collaborate into transmission, becoming a relay that transmits a second stream increasing the transmission rate of the active user and reducing therefore the energy used per bit transmitted (i.e. ECR, see Section 2). A Nash bargaining approach is used to achieve the cooperation assuming that mobile terminals behave selfishly and therefore a motivation is needed. The Nash equilibrium here can be reached if an infinite-stage game is performed. Other publications study the inherent energy-efficient advantages of relaying compared to direct transmission. For instance, the study of [41] conducts an analysis of wireless relays,
Coordinated Multipoint Transmission (CoMP) and the classic BS-MS point-to-point transmission. In the schemes analysed, the BS/relay sleep mode feature is included. From this analysis, some conclusions are drawn: (i) increasing the BS density and reducing the cell size leads to better results in terms of energy efficiency for any of the mechanisms considered, and (ii) wireless relays must have a reduced consumption compared to a BS in order to be an option of being an energy-efficient mechanism. In [42], some transmission mechanisms using direct transmission and relaying are analysed with the additional consideration of having at their disposal the capabilities of average channel state feedback or instantaneous channel state feedback. The results of this article show that cooperative relaying must be used when outage probability target is very low, in order to enhance energy efficiency. On the contrary, for less exigent outage probabilities it is advised to use direct link transmission. In any case the fact of introducing instantaneous channel knowledge features into a transmission mechanism is a key for reduced energy consumption. This latter could be implemented by using the sensing capabilities of the $\mathrm{CR}$, as we will see in Section 5.

In the relay domain, there are still very hot topics still open and a good amount of work to do. The concerns 
on this area for future research are very well expressed in [18]: in order to coordinate the resource allocation for cooperative relaying some extra power is needed, and therefore minimise this overhead energy expended is a task to do. Additionally, this reference remarks the importance of working in bi-directional relaying systems and multi-cell environments, due to most of the work is done for point-to-point transmission and moreover only considering whether uplink or downlink, but not both. Additionally, as we said before in Section 3.1, the reference [29] mentions the introduction of relaying devices as a possibility to enhance a cellular deployment for cell breathing. For such an approach, there are open subjects for real-life implementation such as the coordination mechanisms and the communications protocols that permit the exchange of information to achieve the cooperativity among the different devices [18].

\section{Energy-efficient transmission mechanisms and RRM}

After having studied the CLA techniques, that as we already said, constitute the top layer category of energy saving at network scale, we are going to continue with techniques that can also contribute to energy saving but into a more local reach. We speak of the energy-efficient transmissions mechanisms and the RRM. A first reference dating from 2003, the study of [10] presents a survey of energy-efficient radio techniques. Several mechanisms are shown, such as the transmission power control where the system adapts its power level in function of variables like channel state or transmission peers locations. Also in this reference, some other techniques used for energy-efficient packet transmission are also mentioned: packet size adaptation, FEC/ARQ (forward error correction/automatic repeat request) schemes, adaptive modulation and data rate adaptation and collision avoidance.

If we start discussing about energy efficiency on transmission mechanisms, MIMO systems are extensively reviewed by energy-efficiency surveys like [16-18]. In [16], it is shown the existing relation between data rate, transmission power and energy efficiency for SISO and MIMO systems. The behaviour presented by a SISO system shows that in order to minimise the energy per joule transmitted the system must transmit at very low data rate (i.e. low transmission power). As said in [16] this can be acceptable for some delay tolerant systems such as a sensor network, but for some others like mobile telephony this latter cannot be accepted in order to guarantee a certain level of quality. For MIMO in slow fading channels, it is shown by this paper that higher number of antennas, results in a higher energy efficiency optimal value of the system can be attained. On the other hand, the review of [17] dedicates an important place also to discussing MIMO, mentioning for instance, how MIMO systems combined with adaptive modulation exhibit better energy-efficiency performance as the transmission distance increases compared to classic SISO systems [43].

For OFDMA, the authors of [44] address the energy efficiency for this transmission mechanism in uplink. The combination of OFDMA and the adaptive modulation is studied and some conclusions are provided: (i) higher the number of sub-channels are assigned to a mobile user, higher the attainable energy-efficiency maximum will be for an OFDMA system functioning at an optimal modulation order; (ii) the optimal energy-efficient modulation order decreases with the distance from mobile terminal to the BS. Such results are very useful, however, in the survey given by Li et al. [18] it is remarked that research work has been only focused in the uplink transmission. Future proposals according to [18] must exploit the existing trade-off between energy and spectrum seeking to find an optimal balance between these two variables during system design for OFDMA. A crucial importance is given also in [18] to traffic statistics in order to better allocate resources.

The cooperativity in relaying techniques was already discussed in Section 3. For this section, we will give a brief mention to the cooperation diversity attainable by a scheme of multiple coordinated BSs transmitting to a single user. A comparison of the energy efficiency attainable by CoMP versus wireless relays was already cited in Section 3 [41]. It is shown that due to the spatial diversity introduced by coordinated BSs working cooperatively, the energy efficiency could be enhanced compared to a classic single BS transmission scheme. Similar conclusions can be extracted from [14] where the energy-efficiency performance of different distributed antenna system (DAS) schemes is compared to the classic single BS-MS point-to-point transmission. However, the success of this cooperation and coordination among a group of neighbour BSs relies on backhaul protocols in order to exchange information. Protocols for this purpose are presented in [45].

Another important issue found in the energy-efficiency schemes into literature is related to the RRM. The main idea is to optimise the allocation or utilisation of transmission resources such as bandwidth or time in order to reduce energy consumption. This optimisation problem must be solved under some constraints given by channel conditions, QoS and transmission/receiver system characteristics. The authors of [46] propose the framework of four fundamental trade-offs to be considered among the main resources of a mobile network: deployment costenergy, spectrum-energy, bandwidth-power and delaypower. To find a balance point for such variables not compromising the service quality, availability and network coverage is the goal of RRM approaches. The author of [47] addresses the subject similarly emphasising 
the economic factor by bounding infrastructure costs, energy costs and spectrum costs. This reference studies the behaviour patterns and relations among such variables in order to give a generalised model, very useful for wireless network optimisation regarding the resource trade-offs and cost perspective.

Here we present some examples of proposals in RRM. In such ideas presented, researchers work with the resource trade-offs in order to improve the energy efficiency of a communication system. In [48], for instance, an energyaware admission control and bandwidth allocation mechanism is proposed. This mechanism builds on the fact that for a given curve of ECR (i.e. bits transmitted per joule, see Section 2) versus transmission rate (i.e. bandwidth allocated), the operation points of different mobile terminals for a fixed transmission rate variation, the obtained value of ECR (Joules/bits) does not change in a proportional fixed quantity. Taking advantage of this fact, the article proposes ways for bandwidth allocation/reallocation aiming to optimise energy-efficiency with a minor bandwidth trade-off. Some other references consider the trade-off between transmission time and consumed power. It is shown in [17] that the energy per bit versus time per bit curve has a convex decreasing behaviour. From this point of view, longer the transmission time per bit, higher the energy-efficiency obtainable. However, in a more realistic implementation we consider it exists behind the circuitry energy that increases with transmission time per bit. The combination of both energy components gives a curve where it exists an optimal operation point, which maximises the energy efficiency. Such optimal point should not exceed however the delay constraints that guarantee service quality. Let us present some examples of how the time delay-power trade-off is exploited. In [49], a scheduling algorithm for periodic packets is proposed. As expected, it seeks to optimise the consumed energy by increasing the transmission time. Here, the delay constraint is given by the transmission period of the sequence of periodic packets. Another example is reference [50]. Here, a lazy scheduling mechanism is presented where transmission is buffered respecting delay constraints. It is shown in this article that small-buffered systems exhibit better energy performance compared to non-buffered deterministic schedulers. Last but not least, in the same line of transmission time-consumed power trade-off, the study of [51] proposes a variable-length slot TDMA. Here, the length is chosen by an adaptive mechanism, which makes the decisions based on some other parameters such as the transmission distance and the transmission queue length.

The need of continuously improving the RRM and transmission mechanism is currently concern of mobile communications in order to better exploit and complement the already shown advantages of top layer mechanisms that yield energy efficiency at large scale (i.e. cell breathing, femtocells and relays). In addition, if the transmission techniques and the way in which resources are allocated are improved, a noticeable enhancement will be obtained for the access equipment during active mode, that complements the already important reductions product of sleep modes.

\section{CR}

Joseph Mitola has defined the $\mathrm{CR}$ as "a radio frequency transmitter/receiver that is designed to intelligently detect whether a particular segment of the radio spectrum is currently in use, and to jump into (and out of, if necessary) the temporarily-unused spectrum very rapidly, without interfering with the transmission of other authorised users" [52]. Therefore, a CR system must be capable of reconfiguring their transmission parameters in order to adapt and match the channel conditions [53], and in the same sense we use CR for spectrum optimisation, we can use it in order to optimise energy consumption. On the hardware level, new flexible technologies able to work using different transmission parameters (modulation order, bandwidth, data rate, frequency, power, etc.) and having a wide operational range are needed. Currently, one viable option is the Software Defined Radio (SDR) that compared to other choices like System-on-Chip as mentioned in [53], provides enough flexibility and low cost in a single piece of hardware. The utilisation of CR is a key for enhancing all the set of techniques already discussed. This technique is strongly related to the RRM and transmission mechanisms we analysed due its capability of sensing the channel conditions and make decision on transmission parameters and resources to allocate.

In [54], for instance, a machine-learning mechanism for transmission power assignment in a non-cooperative environment is proposed. A non-cooperative environment is considered given the fact that power strategies among nodes could not be shared in a real scenario (e.g. due to conflict of interests between two operators). Each node chooses the transmission power behaving selfishly, only driven by receiving a reward. Based on the maximisation of this reward, a node can evaluate its strategy without knowing the other nodes strategy. This consideration leads us to an optimal transmission power strategy for all nodes deployed, which converges to Nash equilibrium [54]. In [55], a presentation of applications of CR for green radio systems is carried out. In the examples given, the capability of CR to be aware of surrounding environment is exploited. For instance, CR can help beam-forming control in a multi-sector BS, establishing the radiation pattern shape and tuning the direction of radiated power of sector antennas reducing the energy losses. Another example from the same reference 
presents the possibility of using CR to reduce radiation exposure by alerting the user that the body position towards the associated BS increases cross body exposure.

\section{Component approach}

Research on energy-efficient components is, maybe, one of the most active so far in this domain and without doubts the foundations where benefits of each one of the upper layer techniques discussed are supported. This research includes the internal BS architecture, idle components switch-off and component energy-efficiency enhancement. Probably one of the biggest concerns at this level is the RF power amplifier efficiency and, linked to that, the overall RF transmission chain efficiency. Some new internal BS architectures are being studied, like the one discussed in $[8,14]$, where an amplifier goes right behind each antenna element (on the tower-top), located outside the equipment room. With this architecture the insertion losses due to cable connections are diminished. Those so-called top-tower architectures $[8,14]$ connect the digital section to the RF antenna head-end by optical fibres to minimise here transmission losses. The benefit obtainable from photonics and optical fibres is a very hot topic nowadays and in the future of network research.

As already mentioned, great efforts are being made for the amplifier section, where different architectures and features have been proposed (Class J amplifier [14], switched mode power amplifier-SMPA [56], drain modulation technique [56], etc.). Such proposals promise higher efficiencies compared to pre-distorted Doherty amplifier $[8,22,57]$, which is currently $45-50 \%$. These approaches have the common objective of reducing amplifier losses and increasing power efficiency, linearity and reducing the PAPR. More details on this approach can be found in the above-cited references and in [13]. On the other hand, some manufacturers claim that their state-of-art amplifiers can avoid the use of air-cooling as stated by FUJITSU for its Doherty pre-distorted power amplifier in [57]. This is also true for smaller BS equipment like femto or picoBSs, which do not need an aircooling component as shown in the models presented in [21]. Therefore, avoiding using air-cooling system in larger BS is a clear direction for fabricants. However, it is also possible to take benefit of heat produced by Power Amplifier. The study of [56] shows the work done on benefitting from heating by reutilising the heat generated by using a thermoelectric generator (TEG). Such a device transforms such heat into electricity by allowing some of the dissipated energy to be recovered.

\section{Classification of proposals and synthesis}

In this section, we classify all these mechanisms and proposals for energy-efficient mobile networks that we have discussed so far. In Table 1, we propose a general classification for these proposals. Each technique is assessed in terms of attained energy savings and the consequences of applying such an approach on network planning and operation. Finally, we highlight some of the research challenges for each of these approaches. Then, these mechanisms and proposals can be integrated in the framework model we propose in Figure 8.

In our framework model of classification and integration, we propose a stacked structure, where upper layers need of lower layers to increase their attainable savings. Firstly, we consider the component enhancement as the base of the energy efficiency for the RAN (see Section 6). For this reason, we will name it component baseline layer (CB). The enhancement at this layer permits to relax the design constraint in order to facilitate the design in upper levels. This layer has a critical importance and new developments in internal components must be done for reducing the consumption and mitigate the losses into the BS. As we have seen that a great amount of work is being done around the power amplifier and the effort should continue toward this direction. The reduction of power amplifier consumption reduces the heat dissipated and additionally avoids the necessity of having air-cooling systems into the macroBS, which reduces even more the consumption. Future advances in photonics and optical networks will reduce the losses due to internal components interconnection and at the backhaul level infrastructure.

However, considering the component approach as the solution of all the problems is rather insufficient in order to achieve large-scale savings. A not negligible amount of energy is wasted due to a not fully efficient utilisation of resources, which is even more critical during the idle states of the BS. That is the reason for having upper layers above the components. The next layer is the environment learning and information exchange layer (EL-IE). For the EL-IE layer, we introduce the natural capabilities of CR (see Section 5) to adapt the radio devices transmission parameters based on sensing external conditions. This adaptation will permit to find the optimal transmission strategies that optimise energy efficiency at the RRM level. These CR mechanisms however should not work alone taking into account that also information about surroundings can be complemented by the information shared by BS neighbours. Such information exchange can be done, whether by using the radio link or the backhaul infrastructure in a cooperative approach. The combination of the CR with the cooperative exchange of information among neighbours of a same RAN will permit the BSs systems to have a good picture of environment and channel conditions, even if there are surrounding devices from a different network operator (i.e. non-cooperative neighbours) or any other type of radio devices and services sharing the frequency spectrum. All these efforts 
Table 1 Classification and assessment of the different energy-efficient solutions reviewed

\begin{tabular}{|c|c|c|c|}
\hline $\begin{array}{l}\text { Research } \\
\text { Issue }\end{array}$ & Energy savings/enhancements & $\begin{array}{l}\text { Consequences on planning and } \\
\text { operation stage }\end{array}$ & Challenges \\
\hline $\begin{array}{l}\text { CLA (1/3): cell } \\
\text { breathing and } \\
\text { switching-off } \\
\text { schemes }\end{array}$ & $\begin{array}{l}\text { Energy saving around }(25-50) \% \text { according } \\
\text { to }[25,26,28]\end{array}$ & $\begin{array}{l}\text { Energy efficiency improves with smaller } \\
\text { cells and denser deployments. However, it } \\
\text { increases infrastructure costs }\end{array}$ & $\begin{array}{l}\text { Further work must be done in the } \\
\text { mechanisms that permit the coordination } \\
\text { of distributed/clustered mechanisms at } \\
\text { large-scale networks. This necessity is even } \\
\text { more critical considering that future cell- } \\
\text { breathing mechanism will work in } \\
\text { heterogeneous networks combining } \\
\text { macro/femtocells and relays }\end{array}$ \\
\hline
\end{tabular}

networks

CLA (3/3):

relays

RRM,

mechanisms

and energy-

efficient

transmission

CR

Component approach sleep modes [8]
Results in [41] shows savings of around (520\%) compared to classic single BS-MS point-to-point transmission

There is a diversity of approaches in this domain. Therefore, it is difficult to give a representative figure

CR is a tool that works along with the RRM and the transmission mechanism. It is difficult to give a representative figure
The utilisation of centralised network cellbreathing mechanisms could limit scalability and introduce single point failure nodes

Provides granularity to service provision and coverage by tailoring the type of access device and resources to the real traffic needs.

Operating a macroBS during 1 year can cost 60000 Euros/year, whereas a femtoBS only 200 Euro/year [33].

Careful planning needed: too many femtoBS may imply overprovisioning (if no sleep mode used), increasing unnecessarily the energy consumption [8], i.e. OPEX [34]

The relays are useful as energy-efficient mechanism only if the power consumed by relaying is sufficiently low compared to a direct BS-MS transmission [41,42]

With RRM algorithms and transmission techniques, it exist always the possibility of taking advantage of trade-offs. However, to find the optimal operation point is a complex task

New technologies like SDR [53] are providing flexibility and low cost, which facilitates the introduction of $C R$ in modern communication systems. The $C R$ is a very advantageous tool in environment where it exists conflict of interest and information is not shared among nodes

Having energy-efficient components is the

The main concern is the PA, which nowadays (e.g. Doherty-Pre-distorted, Class $A B)$ has PA eff. around 50\% [22,57]. The Class J amplifier is aimed to achieve PA eff. networks of 85-90\% [14] the SMPA although theoretically could reach $100 \%$ according to [22], so far it has reached PA eff. of $55 \%$ [56]
There are open aspects on coordination and management mechanisms to handle the network heterogeneity: e.g. different levels of resources in function of BS type; different environments, i.e. outdoor, indoor; interference, spectrum management,

The need of protocols and mechanisms in order to have cooperative schemes of relaying is still an open topic to explore. Additionally, further work must be done in bidirectional relaying [18]

Develop new mechanisms considering all different trade-offs is not an easy matter. The task becomes more complex if we consider that future networks are going moving forward to cooperative schemes in these two domains

It must continue the work on flexibility of software and hardware in order to enhance the features that CR may bring to the energy-efficient approaches [53]. It exist open topics to study like the combination of backhauls protocol for information exchange with CR for having communications devices with more accurate information from outside world

Achieve higher components efficiencies. New prototypes like Class J Amplifier [14] and SMPA [56] are candidates for next generation PAs. It is critical for future BSs to have this kind of components. Further work must be done in optical networks and photonics in order to reduce transmission losses at the backhaul and components interconnection are intended to have an instantaneous information feedback that will permit the RRM level to make the best decisions. However, it has a huge importance to have a minimal consumption energy overhead due to these learning and information exchange processes and a lot effort must be done in maintaining here a very low energy regime.
To the benefit of the previous layer we propose an energy-efficient RRM and optimal transmission layer. This layer deals with the resources trade-offs we already spoke in Section 4 for energy-efficient transmission. The resources for transmission are finites and designers must carefully find a balance among the spectrum, the energy, power and time delays. For the current and 


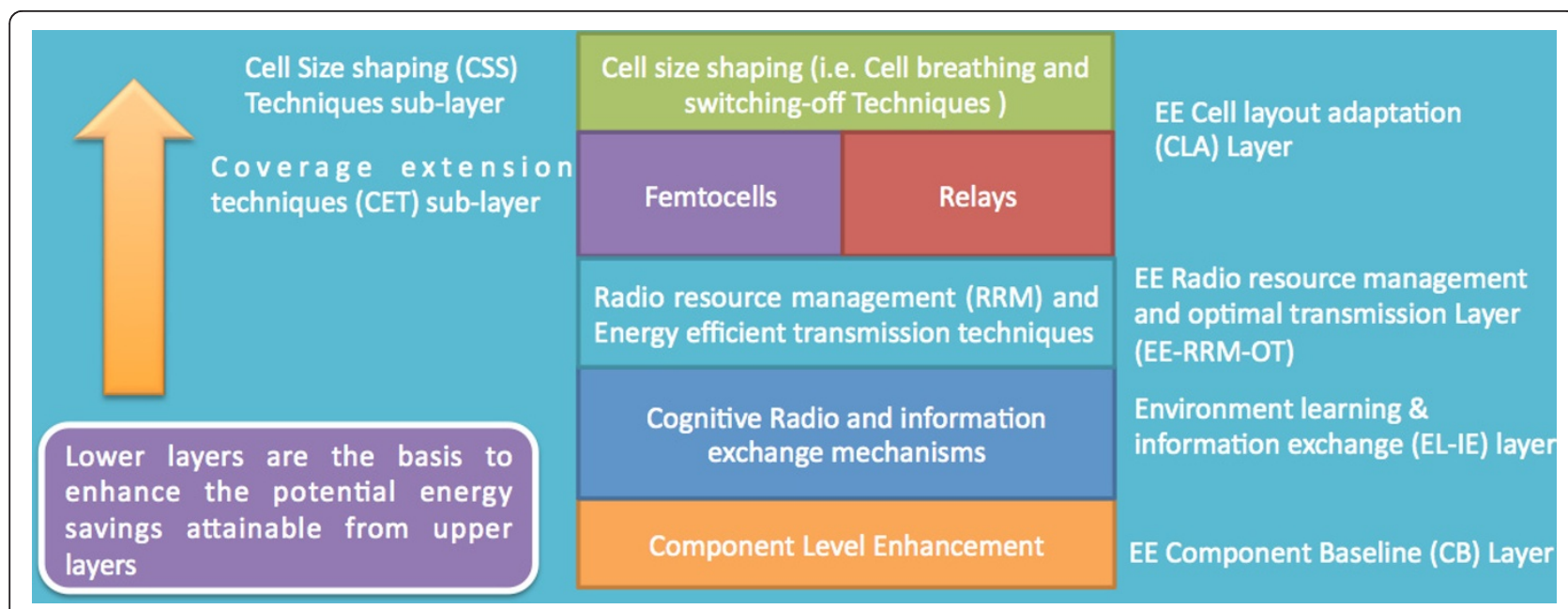

Figure 8 Framework model of energy-efficient approaches

future mobile networks, relax the constraints on transmission time delays is something very difficult, due that such networks are increasing more and more the volume of data traffic for real-time applications like video streaming and multimedia phone calls. This latter makes more exigent the time delay constraints in order to guarantee quality. However, we have seen in Section 4 how frequency and space diversity approaches can help in the increase of the energy efficiency of a system. A clear direction to enhance the transmission performance is to continue the research in techniques like OFDMA and MIMO, which will permit to exploit better their capabilities to reduce the energy per bit figures. Moreover, if we consider that the future of mobile RANs goes toward the hybrid macro-femto approaches and BS cooperative transmissions (e.g. CoMP and DAS) there is a lot of work to do in coordination mechanisms for resource allocation in such approaches. Such RRM mechanisms should additionally permit the coexistence of a cooperative scheme of femto/macro BSs with other elements such as relays, by sharing efficiently the spectrum, avoiding the interference and allocating in a tailored way the resources (e.g. power and spectrum) to serve the incoming users.

Finally, we have the CLA layer. The main mechanisms at this level are the switching-off and cell-breathing schemes that provide savings at a network scale. We put these techniques at the top into the cell size shaping sublayer (CSS). The granularity on service provision and the coverage extension is given by the (CET) sub-layer, where we find femtocells and relays. At the CET sublayer is important to remark the importance of careful planning to avoid femtoBs overprovisioning, as well as, the need of coordination to apply the switching-off and cell breathing not only on the macroBS deployment, but also extend it to the femtoBS and relay level. Here we can say that the main keys to achieve the network scale savings at the CLA layer come from the lower layer approaches: firstly, a better circuitry and components that permit sleep modes with the lowest consumption and rapid transition from sleep to active and vice versa. During active mode the devices must work under power regimes tailored to real service demands by (i) reducing the internal losses and power consumption into the devices, but above all, (ii) by proper choices of resource allocated and adapted transmission parameters. Moreover, a critical point to discuss is the way in which the coordination will be performed in such heterogeneous environment. Although centralised approaches permit to obtain an instantaneous network panoramic, as we discussed in Section 3, is something that limits the scalability and introduce single-point failure nodes into the network. Such coordination mechanism should be therefore a halfway point that as we also said in Section 3 must be implemented by the utilisation of cooperative device clusters. Those devices into a common cluster (e. g. femtoBS, macroBS, relays, etc.) are responsible of an area of coverage with the collective goal of maximising a cluster energy-performance indicator. In order to avoid conflicts with surrounding clusters, to manage the cluster resource allocation and coordinate cooperative transmission by means of heterogeneous devices, the combination of information exchange mechanisms and CR are a clear necessity to obtain an instantaneous information feedback of surroundings, which gives the necessary background information to establish the best actions to take.

\section{Collaborative projects}

Due to the importance of this issue, a number of collaborative projects composed of different organisations 
from different sectors, such as research institutes, universities and telecommunications providers, are dedicated to the energy efficiency in wireless networks. In this section, we summarise those projects.

The GreenTouch consortium [58] is a worldwide largescale project led by Alcatel-Lucent/Bell Labs with partners from academia, research institutes, operators and fabricants, which started in January 2010 and with the duration of 5 years that will finish in January 2015. The ambitious goal intended by GreenTouch is to develop the whole architecture needed to reduce by a factor of 1000 the current levels of energy consumptions in ICT networks. The large-scale proposal of this consortium, as stated in its mission [58], includes the necessary specifications and roadmap to achieve this goal. The projects and axes of GreenTouch cover all the network architecture including the core networking (i.e. switching, routing, transmission, etc.) and the wireless and mobile frontend networks. Their approach can be considered very revolutionary and disruptive. They propose to rethink and reinvent communications systems starting from the principles and only constrained by the law of physics $[15,58]$. So far, we can notice a great interest of GreenTouch in specific areas like optical network and photonics, large-scale antenna systems, dynamic wavelength mechanisms and distributed services [59]. Into GreenTouch we also want to highlight the project GREAT from Huawei [60], specialised in the domain of RRM for energy-efficient networks. An important contribution has been made identifying, analysing and modelling the resource trade-offs in mobile networks as mentioned in Section 4 for the power, energy, spectrum, bandwidth, latency and deployment cost variables $[46,61,62]$.

In Europe, the ICT-EARTH Project (Energy Aware Radio and Network Technologies) into the European Seventh Framework-FP7 is also a very important largescale scale initiative to mention [63]. This project, which is mainly funded by European Commission, started in January 2010 and it is expected to reach its end in June 2012. For the final results of this project, it is expected to reduce, at least by $50 \%$, the energy consumption of mobile networks. ICT-EARTH approaches the energyefficiency problem holistically [15], where all aspects and elements, i.e. social impact, energy efficiency metrics, green network management mechanism, components and green radio transmission aspects, are joined into a common integrated platform [63]. An important contribution of this project is the $E^{3} F$ framework [21] where the network modelling (traffic behaviour, BS energy model, deployment structure, etc.), the necessary energyefficiency performance metrics, the energy-aware RRM techniques which can be applied, the network architecture and extension devices (e.g. femtoBS and relays) are blocks that constitute such approach.
Also in Europe, the OPERA-NET (Optimising Power Efficiency in Mobile Radio Networks) in the framework of CELTIC (Cooperation for a sustained European Leadership in Telecommunications) is also very important project to remark. Led by France Telecom, this project started in June 2008 and with expected end date by May 2011, wanted to respond to the concerns of European Union due to climatic change by enhancing the energy efficiency by $20 \%$ before 2020 [64]. The approach is similar to the one already discussed with ICT-EARTH where the problem is studied from a holistic point of view. Here, different task forces are considered and vital aspects are covered: mobile RAN engineering (e.g. key performance indicators, cell size optimisation, sleep modes, etc.), link level optimisation and technology enablers. About this project, it is to emphasise the important efforts done in the technology enablers' axis, where new power amplifier architectures (e.g. SMPA [22,56]) and other features have been proposed (e.g. drain modulation technique and TEG [56]).

In United Kingdom, the Mobile VCE is a long-term platform started in 1997, which current work stage is the Core 5. This new phase started in January 2009 and duration of 3 years, it is divided into four basic axes: Flexible Networks, User Interaction, Instant Knowledge and Green Radio. The goal in this last work axis is to reduce energy consumption by a factor of 100 in current highspeed data mobile services [65]. A good overview of the work carried out by this project can be seen in $[7,14,66]$. So far, the project has presented some important contributions [65] like the random network coding as a better energy-efficient alternative to the classic Hybrid ARQ (HARQ) [67], or the fibre-to-air concept where the RF signals are transported via optical fibre to the antenna transmitter, reducing therefore the transmission line losses $[14,68]$. However, the most remarkable contribution could be the introduction of the class J amplifier that promises efficiencies between $85-90 \%[14,69]$.

On the other hand, in Germany, the Cool Silicon Cluster of Excellence, a project led by Technical University of Dresden, is a research effort mainly devoted to microelectronics advances on energy-efficient components in the ICT domain [70]. In the specific area of green communications systems, there is an important set of topics being studied right now by Cool Silicon. There exists, for instance, a great interest from this initiative in developing mobile electronic devices with reduced consumption powered by solar energy. This latter is also considered for femto/macroBSs where low regime consumption is expected by optimising not only internal component functioning, but also computing resources utilisation. Moreover, the Cool Silicon cluster is doing also research on topics like energy-efficient relays supported on the latest VLSI techniques, software optimisation for vehicular 
applications, and others like the study of transition from analogue technologies to low-consumption digital radio [70].

There exist other initiatives like the GREENET, a training program to prepare future PhDs in Energy Efficiency for Green mobile networks led by the Polytechnic University of Catalonia and supported by European Commission, the FP7 and the 2010 Marie Curie Programme [71]. The project that started in 2011 with duration of 3 years studies the energy efficiency at different levels into a collaborative scheme and a structured training platform with teams working on different approaches: physical layer components, energy-aware RRM and MAC mechanisms, network coding and cooperative schemes [71,72].

In the area of femtocells, The BeFemto Project, started in January 2010 with an expected duration of 30 months, is fully dedicated to femtocells technology and its real applicability in mobile networks [73]. Within the goals of this project, it is intended to reduce the maximum averaged power under the $10 \mathrm{~mW}$ for indoor femtoBSs, and reach a system spectral efficiency of at least $8 \mathrm{~b} / \mathrm{s} / \mathrm{Hz} /$ cell. Other objectives propose to find new uses and application of femtocells in different environments considering additional aspects like mobility (e.g. vehicular applications). Some interesting publications have come out from this project in the domain of femtocell networks, like for [74] power allocation and [75] for interference management.

Finally, we briefly talk the project eWIN (energy-efficient wireless networking) led by KTH in Sweden[76]. The project proposes to provide the fundamental basis that permit to reduce the energy consumption, as well as to rethink the way as wireless technology architectures are conceived and designed, which so far only takes care of elements like availability, coverage and throughput capacity. The approach of the project studies specifically the RRM optimisation, the protocols and mechanisms, in order to deal with the resource tradeoffs, which are the key enablers of future energy-efficient architectures. A good example of the work being performed in this project is the study of [47].

\section{Conclusion}

This article intended to provide an up-to-date overview on green wireless networks research. We described each of the main global approaches used, presenting some references for each of them. We proposed a classification of the main research axes, analysing the expected enhancements, the consequences and the challenges of each one of those approaches. Clearly, none of these trends is sufficient in itself and a combined approach is necessary as presented in our classification and integration model. A lot of additional hard work is needed in each one of the presented layers in order to reach the important goals of energy reduction expected in the years to come.

We think that future research in this topic should address the minimisation of energy consumption by combining more than one of these approaches. Other perspectives can also be included such as the economic and cost considerations.

As in many current research topics, practical implementations, in the case of green wireless networks, are needed quickly. An important work is needed in order to make the link between advanced research proposals (most of them yet to be carried out) and practical mechanisms that can be implemented by operators.

\section{Acknowledgements}

The authors wish to thank Patrick McLaughlin for his helpful comments.

\section{Competing interests}

The authors declare that they have no competing interests.

Received: 30 September 2011 Accepted: 13 April 2012

Published: 13 April 2012

\section{References}

1. Gartner, Green IT The New Industry Shockwave, in Symposium/ITXPO conference (April 2007)

2. GeSI, Global e-sustainability initiative: SMART 2020: Enabling the low carbon economy in the information age. (2008)

3. U.S. Residential Information Technology Energy Consumption in 2005 and 2010, Final report, prepared by TIAX LLC for U.S. Department of Energy (March 2006)

4. B Gorjni, ETSI work programme on energy saving, in INTELEC 2007. Rome, Italy, 174-181 (September 2007)

5. Road map to reduce energy consumption, Green Telco World Congress (2009)

6. U Barth, Alcatel-Lucent, Bell Labs Stuttgart, How To Reduce-Green House Gas Emissions From ICT Equipment, (Slides) Wireless Networks, EARTH research project, ETSI Green Agenda (Nov 2009)

7. PJ Chung, Green Radio-the case for more efficient cellular base stations, (slides) UK-Taiwan ICT Workshop: Smart \& Green Communications, (University of Taiwan March 2010).

8. H Claussen, LTW Ho, F Privit, Effects of joint macrocell and residential picocell deployment on the network energy efficiency, in Proc of PIMRC 2008. Cannes, France, 1-6 (September 2008)

9. Alcatel-Lucent and Vodafone Chair on Mobile Communication Systems, Study on Energy Efficient Radio Access Network (EERAN) Technologies, (Unpublished Project Report, Technical University of Dresden, Dresden, Germany 2009).

10. H Karl, An overview of energy-efficiency techniques for mobile communication systems. Technical Report, Telecommunication Networks Group, (Technical University Berlin (TKN) September 2003)

11. D Ferling, T Bohn, D Zeller, P Frenger, I Godor, Y Jading, W Tomaselli, Energy efficiency approaches for radio nodes, in Proc of Future Net Mobile Summit '10. Florence, Italy, 1-9 (June 2010)

12. CE Jones, KM Sivalingam, P Agrawal, J Chen Cheng, A survey of energy efficient network protocols for wireless networks. ACM Wirel Netw J. 7(4), 343-358 (2001)

13. LM Correia, D Zeller, O Blume, D Ferling, Y Jading, I Godor, G Auer, L Van Der Perre, Challenges and enabling technologies for energy aware mobile radio networks. IEEE Commun Mag. 48(11), 66-72 (2010)

14. JS Thompson, Congzheng Han, T Harrold, T Armour, I Krikidis, S Videv, PM Grant, H Haas, I Ku, Cheng-Xiang Wang, Tuan LeAnh, MR Nakhai, Jiayi Zhang, L Hanzo, Green Radio: radio techniques to enable energy efficient wireless networks. IEEE Commun Mag (Special Issue: Green

Communications). 49(6), 46-54 (2010) 
15. O Blume, D Zeller, U Barth, Approaches to energy efficient wireless access networks. in Proc of ISCCSP 2010. Limassol, Cyprus 1-5 (March 2010)

16. EV Belmega, S Lasaulce, M Debbah, A survey on energy-efficient communications, in PIMRC 2010 Workshop. Istambul, Turkey, 289-294 (2010)

17. G Miao, N Himayat, GY Li, Cross-layer optimization for energy-efficient wireless communications: a survey. Wirel Commun Mob Comput. 9(4), 529-542 (2009)

18. GY Li Zhikun Xu, Cong Xiong, Chenyang Yang, Shunging Zhang, Yan Chen, Shugong $\mathrm{Xu}$, Energy-efficient wireless communications: tutorial, survey, and open issues. IEEE Wirel Commun. 18(6), 28-35 (2011)

19. R Bolla, R Bruschi, F Davoli, F Cucchietti, Energy eficiency in the future Internet: a survey of existing approaches and trends in energy-aware fixed networks infrastructures. IEEE Commun Surv Tutor. 13(2), 223-244 (2011)

20. Tao Chen, Haesik Kim, Yang Yang, Energy efficiency metrics for green wireless communications, in Conf Wireless Communications and Signal Processing (WSCP). Suzhou, China, 1-6 (Oct 2010)

21. EARTH, INFSO-ICT-247733 EARTH, Energy efficiency analysis of the reference systems, areas of improvements and target breakdown (March 2010)

22. Opera-Net Project: Optimising Power Efficiency in mobile Radio Networks, (Slides) Celtic Event, Paris, France (March 2009)

23. Liqiang Zhao, Jian Cai, Hailin Zhang, Radio-efficient adaptative modulation and coding: green communication perspective, in VTC Spring 2011. Budapest, Hungary, 1-5 (May 2011)

24. Energy Efficiency of Wireless Access Network Equipment, ETSI TS 102 706, 2009 http://www.etsi.org

25. L Chiaraviglio, D Ciullo, M Meo, MA Marsan, Energy aware UMTS access networks. in W-GREEN 2008. Lapland, Finland (September 2008)

26. MA Marsan, L Chiaraviglio, D Ciullo, M Meo, Optimal energy savings in cellular access networks, in Proc ICC Workshops 2009. Dresden, Germany, 1-5 (June 2009)

27. Sheng Zhou, Jie Gong, Zexi Yang, Zhisheng Niu, Peng Yang, Green mobile access network with dynamic base station energy saving. in IEICE, Tech Rep. 109(262), 25-29 (Oct 2009)

28. Zhisheng Niu, Yiqun Wu, Jie Gong, Zexi Yang, Cell zooming for costefficient green cellular networks. IEEE Commun Mag. 48(11), 74-79 (2010)

29. Xiangnan Weng, Dongxu Cao, Zhisheng Niu, Energy-efficient cellular network planning under insufficient cell zooming, in VTC 2011. Budapest, Hungary, 1-5 (May 2011)

30. G Micallef, P Mogensen, H Scheck, Cell size breathing and possibilities to introduce cell sleep mode, in European Wireless 2010. Lucca, Italy, 111-115 (April 2010)

31. MF Hossain, KS Munasinghe, A Jamalipour, An eco-inspired energy efficient access network architecture for next generation cellular systems, in IEEE WCNC 2011. Cancun, México, 992-997 (March 2011)

32. A Conte, A Feki, L Chiaraviglio, D Ciullo, M Meo, MA Marsan, Cell wilting and blossoming for energy efficiency. IEEE Wirel Commun. 18(5), 50-57 (2011)

33. V Chandrasekhar, JG Andrews, AA Gatherer, Femtocell networks: a survey. IEEE Commun Mag. 46(9), 59-67 (2008)

34. Analysys: Picocells and Femtocells: Will Indoor Base Stations Transform the Telecoms Industry? http://www.analysysmason.com/About-Us/News/Insight/ Picocells-and-femtocells-could-dramatically-alter-the-mobile-landscape/? ReturnUrl=http\%3A/www.analysysmason.com/Search/\%3Fquerytype\% 3DAnyWords\%26page\%3D1\%26perpage\%3D10\%26query\%3Dhttp\%3A// www.analysysmason.com/RPicocells+and+femtocells+will+indoor+base +stations+transform+the+telecoms+industry\%26new_search\%3Dtrue\% 26sortby\%3DRelevance\%23\%21/_Search_Filter_\%3Fquery\%3Dhttp\% 3A___ www.analysysmason.com_RPicocells+and+femtocells+will+indoor +base+stations+transform+the+telecoms-industry\%26featuredltemsCount\% 3D0\%26back\%3Dtrue

35. G Micaleff, P Mogensen, HO Scheck, E Lang, Energy efficient evolution of mobile networks macro-only upgrades vs. a joint-pico deployment strategy, in Conf VTC (Spring) 2011. Budapest, Hungary, 1-5 (May 2011)

36. I Güvenc, Moo-Ryong Jeong, F Watanabe, H Inamura, A hybrid frequency assignment for femtocells and coverage area analysis for co-channel operation. IEEE Commun Lett. 12(12), 880-882 (2008)

37. Yong Bai, Juejia Zhou, Lan Chen, Hybrid spectrum usage for overlaying LTE macrocell and femtocell, in Proc IEEE Globecom 2009. Hawaii, USA, 1-6 (November 2009)

38. D Ezri, S Shilo, Green cellular-optimizing the cellular network for minimal emission from mobile stations, in IEEE International Conference on
Microwaves, Communications, Antennas and Electronics Systems COMCAS. Tel Aviv, Israel, 1-5 (March 2009)

39. C Comaniciu, N Mandayam, HV Poor, Radio resource management for green wireless networks, in Conf IEEE VTC (Fall) 2009. Alaska, USA, 1-5 (September 2009)

40. M Nokleby, B Aazhang, User cooperation for energy-efficient cellular communications, in IEEE International Conference on Communications (ICC), 2010. Cape Town, South Africa, 1-5 (May 2010)

41. Dongxu Cao, Sheng Zhou, Chao Zhang, Zhisheng Niu, Energy saving performance comparison of coordinated multi-point transmission and wireless relaying, in Proc Globecom 2010. Miami, FL, USA, 1-5 (December 2010)

42. I Krikidis, JS Thompson, PM Grant, Cooperative relaying with feedback for lifetime maximization, in Proc IEEE ICC2010. Cape Town, South Africa, 1-6 (May 2010)

43. Shuguang Cui, AJ Goldsmith, A Bahai, Energy-efficiency of mimo and cooperative mimo techniques in sensor networks. IEEE J Sel Areas Commun. 22(6), 1089-1098 (2004)

44. Guowang Miao, N Himayat, Ye Li, Energy-efficient design in wireless OFDMA, in Proceedings of IEEE International Conference on Communications, 2008, ICC '08. Beijing, China, pp. 3307-3312 (2008)

45. TA Le, MR Nakhai, Throughput analysis of network coding enable wireless backhauls. IET Commun. 5(10), 1318-1327 (2011)

46. Yan Chen, Shunqing Zhang, Shugong Xu, GY Li, Fundamental trade-offs on green wireless networks. IEEE Commun Mag. 49(6), 30-37 (2011)

47. J Zander, Energy and cost efficient ultra-high capacity wireless access, in Greenet Workshop (Slides), IEEE VTC Spring 2011. Budapest, Hungary (May 2011)

48. Xinbing Wang, Wenye Wang, Energy-aware call admission control scheme in wireless cellular networks, in Conf Globecom 2004. Dallas, TX. 3 , 1570-1574 (November 2004)

49. Xiao Qin, M Alghamdi, M Nijim, Ziliang Zong, K Bellam, Scheduling of periodic packets in energy-aware wireless networks, in Conf IPCCC 2007. New Orleans, USA, 210-217 (April 2007)

50. E Uysal-Biyikoglu, B Prabhakar, A El Gamal, Energy-efficient packet transmission over a wireless link. IEEE/ACM Trans Netw. 10(4), 487-499 (2002)

51. Shuguang Cui, A Goldsmith, A Bahai, Joint modulation and multiple access optimization under energy constraints, in Proc IEEE Globecom 2004. Dallas, TX, USA, 151-155 (November 2004)

52. B Fette, Three obstacles to cognitive radio, EE Times design, August 2004 http://www.eetimes.com/design/other/4003526/Three-obstacles-tocognitive-radio

53. A Dejonghe, B Bougard, S Pollin, J Craninckx, A Bourdoux, L Ven der Perre, $F$ Catthoor, Green reconfigurable radio systems. IEEE Signal Process Mag. 24(3), 90-101 (2007)

54. Xianfu Chen, Zhifeng Zhao, Honggang Zhang, Green transmit power assignment for cognitive radio networks by applying multi-agent Qlearning approach, in European Wireless Technology Conference 2010. France, 113-116 (September 2010)

55. J Palicot, Cognitive radio: an enabling technology for the green radio communications concept. IWCMC'09. Leipzig, Germany 489-494 (June 2009)

56. Opera-Net project stand No. 42, NEM Summit 2010, Optimising power efficiency in mobile radio networks, (Slides) Barcelona, Spain. (October 2010)

57. S Vadgama, Trends in green wireless access. Pub FUJITSU Sci Technol J. 45(4), 404-408 (2009)

58. GreenTouch web page http://www.greentouch.org/index.php?page=aboutus

59. Green Touch Press Release, Green Touch Demonstrates First Technology On Road to Realizing 1,000-Fold Network Energy Efficiency Vision, London, UK. (Feb 2011)

60. Yan Chen, Great project overview, (Slides) Green Touch open Forum, Seoul, Korea. (April 2011)

61. Yan Chen, Shunqing Zhang, Shugong Xu, Characterizing energy efficiency and deployment efficiency relations for green architecture design, in Proc IEEE ICC E2NET Workshop. Cape Town, South Africa, 1-5 (June 2010)

62. Y Chen, S Zhang, S Xu, Impact of non-ideal efficiency on bits per joule performance of base station transmissions, in Proc IEEE VTC Spring. Budapest, Hungary, 1-5 (June 2011) 
63. ICT EARTH web page https://www.ict-earth.eu

64. Opera-Net web page http://opera-net.org/default.aspx

65. Mobile VCE web page http://www.mobilevce.com

66. P Grant, Green radio - the case for more efficient cellular base stations, (Slides) Mobile VCE, Globecom (2010)

67. Mobile VCE, Random Network Coding for multimedia streaming in LTE-A http://www.mobilevce.com/newsite/sites/default/files/infostore/ibrief_RAND \%20NET\%287\%29.pdf

68. Mobile VCE, Fibre-to-Air for Cellular Applications-Improving Network Flexibility \& Energy Efficiency http://www.mobilevce.com/newsite/sites/ default/files/infostore/ibrief_FIBREtoAIR\%284\%29.pdf

69. Mobile VCE, Power Amplifiers for $4 G$ and beyond- managing the efficiency, bandwidth and linearity tradeoff http://www.mobilevce.com/newsite/sites/ default/files/infostore/ibrief_3G\%20WiFi\%285\%29.pdf

70. Cool-Silicon web page http://www.cool-silicon.org/

71. GREENET web page http://www.fp7-greenet.eu/

72. M Di Renzo, L Alonso, FHP Fitzek, A Foglar, F Granelli, F Graziolsky, C Grueut, H Haas, G Kormentzas, Al Perez, J Rodriguez, J Thompson, C Verikoukis, GREENET_-an early stage training network in enabling technologies for green radio, in 2nd Green Wireless Communications and Networks Workshop, IEEE VTC 2011. Budapest, Hungary, 1-5 (2011)

73. BeFemto web page http://www.ict-befemto.eu/

74. C Abgrall, EC Strinati, JC Belfiore, Distributed power allocation for interference limited networks, in PIMRC 2010. Istambul, Turkey, 1342-1347 (2010)

75. CHM de Lima, M Bennis, K Ghaboosi, M Latva-aho, Interference management for self-organized femtocells towards green networks, in PIMRC Workshops. Istambul, Turkey, 352-356 (September 2010)

76. eWIN web page http://www.wireless.kth.se/research/projects/19-ewin

doi:10.1186/1687-1499-2012-142

Cite this article as: Suarez et al: An overview and classification of research approaches in green wireless networks. EURASIP Journal on Wireless Communications and Networking 2012 2012:142.

\section{Submit your manuscript to a SpringerOpen ${ }^{\circ}$ journal and benefit from:}

- Convenient online submission

- Rigorous peer review

- Immediate publication on acceptance

- Open access: articles freely available online

- High visibility within the field

- Retaining the copyright to your article

Submit your next manuscript at $\gg$ springeropen.com 